\title{
PÉNÉTRATION DES AEDES DANS LES MAISONS
}

\author{
Par J. CALLOT et C. VERMEIL.
}

Lavier et Dao Van Ty (1944) ont attiré l'attention sur le comportement anormal de certaines espèces d'Aedes exophiles pendant l'été de 1943. C'est ainsi qu'ils ont vu Aedes cantans et Aedes communis pénétrer dans les maisons pour y piquer, non seulement au rez-dechaussée, mais aussi au premier étage. Ils rappellent, à titre de comparaison avec ces observations faites aux environs d'Etampes, qu'à la station de Richelieu, on a constaté la pénétration et l'agression de cantans, annulipes et geniculatus, mais toujours très restreintes.

Lavier et Dao Van Ty expliquent ce comportement anormal de cantans et de communis par la sécheresse très spéciale de 1943. Dans la discussion qui suivit cette communication, E. Roubaud fit remarquer qu'en Vendée, Aedes caspius pénètre souvent aux rez-dechaussée pour attaquer l'homme et il attribue ce fait à l'humidité atmosphérique. Nous avons constaté la même agressivité de caspius dans les maisons en Camargue et Marshall la signale en Angleterre dans des régions où ce moustique abonde.

On voit que l'accord n'est pas fait sur les raisons qui poussent les Aedes à pénétrer pour piquer et, dans ces observations, il s'agit toujours de femelles.

L'Aedes vexans, qui constitue un véritable fléau dans les régions où il pullule, est le plus souvent donné comme attaquant l'homme à l'extérieur. Toussaint, dans sa brochure sur les moustiques d'Alsace (1939), parle surtout de ses méfaits à l'extérieur et ne signale qu'incidemment qu'il entre dans les maisons et surtout dans les écuries. Roman, dans une publication sur les moustiques fluviatiles de la région de Lyon (1938), donne vexans comme agressif seulement à l'extérieur.

En réalité, tout dépend de l'abondance de vexans. En Alsace, lors des années de développement normal, si l'on peut dire, et $\dot{a}$ fortiori dans les années sèches, comme 1947, le "fléau des moustiques 》 ne se manifeste que peu ou pas, du moins en ville, et seul l'entomo-

Ann, de Parasitologie, T. XXIII, $x^{\circ 5} 5-6 .-1948$, p. 334-336. 
logiste se préoccupe de ces diptères. Cependant, et même dans les années "sans moustiques ", on peut constater que des Aedes vexans pénètrent dans les maisons, mais ils attaquent seulement au rez-de-chaussée, dans des logements situés à proximité de leurs abris diurnes (arbres bas, buissons, massifs). La pénétration se fait au moment du coucher du soleil, ils attaquent, se repaissent, puis disparaissent.

Lors des années ou des périodes " à moustiques 》, comme ce fut le cas en juillet 1948 , à la suite d'une crue d'été du Rhin, la multiplication des $A$. vexans est invraisemblable : ils envahissent les villes et $\mathrm{y}$ attaquent avec acharnement. Ils pénètrent dans les maisons, non seulement aux rez-de-chaussée, mais leur marée gagne le premier étage et parfois le second ; mais surtout dans des maisons situées à proximité des gîtes diurnes.

Alors qu'à l'extérieur, ils sont agressifs à l'ombre toute la journée, à l'intérieur, c'est à partir du coucher du soleil et pendant le début de la nuit, puis un peu au matin, qu'ils sont les plus nombreux et le plus agressifs.

Malgré leur abondance, ils ne séjournent que peu dans les maisons et, en tout cas, on n'en voit jamais de gorgés. Par contre, dans les clapiers et les écuries, qu'il s'agisse d'année à moustiques ou d'année sans moustiques, les femelles gorgées de vexans sont extrêmement nombreuses sur les murs et les plafonds; on peut les compter par milliers dans les écuries.

Tout ceci est de constatation aisée et l'on s'étonne qu'à Strasbourg, les services préposés à la lutte contre les moustiques attribuent au seul Culex pipiens Linné les attaques dont les habitants souffrent dans les maisons (1).

Mais si les femelles d'Aedes pénètrent dans les maisons, il est aussi fréquent d'y rencontrer des mâles. Les mâles d'Aedes vexans suivent les femelles, comme le fait a déjà été signalé, ou, du moins, se déplacent assez loin du gite larvaire, et on en rencontre à Strasbourg en pleine ville, dans les mêmes abris diurnes que les femelles, en des points situés à trois kilomètres au moins des lieux d'éclosion. Ils pénètrent volontiers dans les maisons, aussi bien le jour que la nuit ; la nuit, ils semblent être légèrement attirés par la lumière.

(1) Culex autogenicus Roubaud existe à Strasbourg. mais y est rare, étant donnćes les cenditions précaires qu'il peut $\mathbf{y}$ trouver. Et, du reste, on peut remarquer, sans détermination entomologique, qu'il ne s'agit pas de moustiques domestiques dans la « plaie » des moustiques. Les invasions ont toujours lieu à des moments assez précis, correspondant, soit à des inondations volontaires de prairies, soit à des crues du Rhin. On ne voit pas bien quelle pourait être l'influence de ces facteurs sur la multiplication d'un moustique tel que l'autogenicus. 
Leur abondance est parfois considérable par rapport aux femelles et l'examen entomologique prouve que d'autres mâles que ceux de vexans pénètrent aussi. C'est ainsi que le 28 juillet 1948 , à 13 h. 30 , l'examen de 43 moustiques, ramassés au hasard après la pulvérisation d'un insecticide dans une pièce, montre qu'il s'agit de 16 femelles de vexans, de 25 mâles de vexans, un mâle de sticticus et un mâle de cantans, c'est-à-dire des moustiques les plus communs après le grand développement de cette époque.

Il a été impossible d'établir un rapport entre l'état de l'atmosphère (température, humidité) et la présence ou l'absence d'Aedes vexans dans les maisons. Par contre, il y a un rapport très net entre leur abondance et la pénétration. Au moment de la poussée qui suit les éclosions massives, ils sont fréquents dans les maisons, puis cela décroît, et enfin on n'en voit plus que quelques individus.

\section{- RÉSumÉ}

En conclusion, nous attirons l'attention sur ce fait que les Aedes, considérés en général comme des moustiques surtout agressifs à l'extérieur, peuvent parfaitement, et en grand nombre, pénétrer dans les maisons au moment des grandes poussées de développement. Les femelles s'y montrent agressives. Elles sont souvent accompagnées par des mâles.

\section{BIBLIOGRAPHIE}

Lavier (G.) et Dao van Ty. - Comportement anormal de certains Edes pendant l'été de 1943. Bull. Soc. Path. Exot., XXXVII, 1944, p. 245.

Roman (E.). - Sur la biologie des moutiques fluviatiles, Avenir Méd., août 1938. Toussaint (A.). - La lutle contre les moustiques dans le département du BasRhin, Strasbourg, 1939.

Instıtut de Parasitologie, Faculté de Médecine, Strasbourg (Directeur : Prof. J. Callot) 